\title{
Upper Bound on the Dark Matter Total Annihilation Cross Section
}

\author{
John F. Beacom, ${ }^{1,2,3}$ Nicole F. Bell, ${ }^{4,5}$ and Gregory D. Mack ${ }^{1,3}$ \\ ${ }^{1}$ Department of Physics, The Ohio State University, Columbus, Ohio 43210, USA \\ ${ }^{2}$ Department of Astronomy, The Ohio State University, Columbus, Ohio 43210, USA \\ ${ }^{3}$ Center for Cosmology and Astro-Particle Physics, The Ohio State University, Columbus, Ohio 43210, USA \\ ${ }^{4}$ California Institute of Technology, Pasadena, California 91125, USA \\ ${ }^{5}$ School of Physics, The University of Melbourne, Victoria 3010, Australia \\ (Received 4 August 2006; revised manuscript received 17 September 2007; published 5 December 2007)
}

\begin{abstract}
We consider dark matter annihilation into standard model particles and show that the least detectable final states, namely, neutrinos, define an upper bound on the total cross section. Calculating the cosmic diffuse neutrino signal, and comparing it to the measured terrestrial atmospheric neutrino background, we derive a strong and general bound. This can be evaded if the annihilation products are dominantly new and truly invisible particles. Our bound is much stronger than the unitarity bound at the most interesting masses, shows that dark matter halos cannot be significantly modified by annihilations, and can be improved by a factor of 10-100 with existing neutrino experiments.
\end{abstract}

DOI: 10.1103/PhysRevLett.99.231301

The self-annihilation cross section is a fundamental property of dark matter. For thermal relics, it sets the dark matter mass density, $\Omega_{\mathrm{DM}} \sim 0.3$, and, in these and more general nonthermal scenarios, also the annihilation rate in gravitationally collapsed dark matter halos today [1]. How large can the dark matter annihilation cross section be? There are two general constraints that bound the rate of dark matter disappearance. (Throughout, we mean the cross section averaged over the halo velocity distribution, i.e., $\left\langle\sigma_{A} v\right\rangle$, where $v_{\text {rms }} \sim 10^{-3} c$.)

The first is the unitarity bound, developed for the earlyuniverse case by Griest and Kamionkowski [2], and for the late-universe halo case by Hui [3]. In the plane of $\left\langle\sigma_{A} v\right\rangle$ and dark matter mass $m_{\chi}$, this allows only the region below a line $\left\langle\sigma_{A} v\right\rangle \sim 1 / m_{\chi}^{2}$ (this will be made more precise below). The second is provided by the model of Kaplinghat, Knox, and Turner (KKT) [4], in which significant dark matter annihilation is invoked to resolve a conflict between predicted (sharp cusps) and observed (flat cores) halo profiles. Since this tension may have been relaxed [1], we reinterpret this type of model as an upper bound, allowing only the region below a line $\left\langle\sigma_{A} v\right\rangle \sim m_{\chi}$. That the KKT model requires $\left\langle\sigma_{A} v\right\rangle$ values $\gtrsim 10^{7}$ times larger than the natural scale for a thermal relic highlights the weakness of the unitarity bound in the interesting $\mathrm{GeV}$ range. However, there have been no other strong and general bounds to improve upon these.

While these bound the disappearance rate of dark matter, they say nothing about the appearance rate of annihilation products, instead assuming that they can be made undetectable. To evade astrophysical limits, the branching ratios to specific final states can be adjusted in modeldependent ways. However, a model-independent fact is that the branching ratios for all final states must sum to $100 \%$. A reasonable assumption is that these final states are
PACS numbers: 95.35.+d, 98.62.Gq, 98.70.Vc, 95.85.Ry

standard model (SM) particles. We show that the most difficult SM final state to detect is neutrinos; that surprisingly strong flux limits can be simply derived from recent high-statistics data; and that we may interpret these as bounding all SM final states, and hence the dark matter total annihilation cross section. See Fig. 1.

If dark matter is not its own antiparticle and if there is a large particle-antiparticle asymmetry, then annihilation could be prohibited, making all bounds inapplicable or irrelevant. Our bound can be evaded if the final states are dominantly new and truly invisible non-SM particles, in which case all dark matter annihilation searches will be more challenging; we quantify an upper bound on the branching ratio to SM final states below.

Probing dark matter disappearance. - For dark matter that is a thermal relic, the cross section required to ensure $\Omega_{\mathrm{DM}} \sim 0.3$ is $\left\langle\sigma_{A} v\right\rangle \sim 3 \times 10^{-26} \mathrm{~cm}^{3} \mathrm{~s}^{-1}$ [1]. KKT discussed several models in which the dark matter is not a thermal relic; e.g., it might have acquired mass only in the late universe, or have been produced through the late decays of heavier particles [4]. As emphasized in Refs. [3,4], it is interesting to directly ask how large the annihilation cross section could be in halos today, irrespective of possible early-universe constraints.

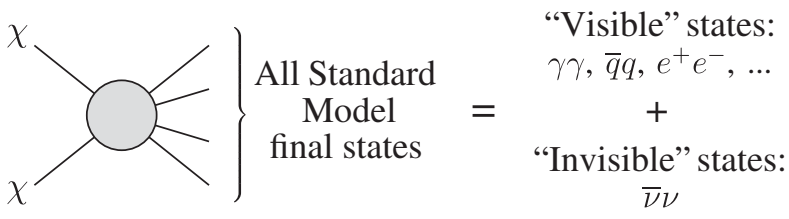

FIG. 1. Annihilation of dark matter into SM final states. Since all final states except neutrinos produce gamma rays (see text), we can bound the total cross section from the neutrino signal limit, i.e., assuming $\operatorname{Br}$ ("Invisible") $\simeq 100 \%$. 
Unitarity sets a general upper bound on $\left\langle\sigma_{A} v\right\rangle$, and can be evaded only in certain unusual cases $[2,3,5]$. In the lowvelocity limit where the cross section is assumed to be $s$-wave dominated, $\left\langle\sigma_{A} v\right\rangle \leq 4 \pi / m_{\chi}^{2} v$, or

$$
\left\langle\sigma_{A} v\right\rangle \leq 1.5 \times 10^{-13} \frac{\mathrm{cm}^{3}}{\mathrm{~s}}\left[\frac{\mathrm{GeV}}{m_{\chi}}\right]^{2}\left[\frac{300 \mathrm{~km} / \mathrm{s}}{v_{\mathrm{rms}}}\right] .
$$

In the KKT model, the required cross section to sufficiently distort the dark matter profiles of galaxies is

$$
\left\langle\sigma_{A} v\right\rangle_{\mathrm{KKT}} \simeq 3 \times 10^{-19} \frac{\mathrm{cm}^{3}}{\mathrm{~s}}\left[\frac{m_{\chi}}{\mathrm{GeV}}\right] .
$$

(Similar effects are attained via elastic interactions [6]. A large self-annihilation cross section implies a large elastic self-scattering cross section, but not vice versa [3].) Hui [3] showed that unitarity restricts the KKT model to relatively small masses; for $v_{\mathrm{rms}}=300 \mathrm{~km} / \mathrm{s}, m_{\chi} \lesssim 80 \mathrm{GeV}$. There have been no other model-independent methods to constrain the KKT model. We argue next that dark matter disappearance must be accompanied by the appearance of something, and the bound on the weakest final-state bounds all of them. The appearance rate bounds $\left\langle\sigma_{A} v\right\rangle$ directly, independent of which partial waves dominate $\sigma_{A}$, i.e., its $v$ dependence.

Revealing neutrino appearance. - We assume that annihilation proceeds to SM particles, and express the cross section in terms of branching ratios to "visible" and "invisible" final states, such as gamma rays and neutrinos, respectively, as in Fig. 1. If the branching ratio to a specific final state were known, then a bound on that appearance rate would yield a bound on the total cross section, inversely proportional to this branching ratio. However, the branching ratios are model dependent, and any specific one can be made very small, making that bound on $\left\langle\sigma_{A} v\right\rangle$ very weak, e.g., for $m_{\chi}=1 \mathrm{GeV}$, KKT require $\operatorname{Br}(\gamma) \lesssim 10^{-10}$ to allow their total cross section [4]. Note that gamma-ray data constrain only the product $\left\langle\sigma_{A} v\right\rangle \operatorname{Br}(\gamma)$, and the bounds vary with $m_{\chi}$.

KKT [4] and Hui [3] assume invisible but unspecified final states. It is clear that most SM final states produce gamma rays. Quarks and gluons hadronize, producing pions, where $\pi^{0} \rightarrow \gamma \gamma$; the decays of weak bosons and tau leptons also produce $\pi^{0}$. The stable final state $e^{+} e^{-}$is not invisible, since it produces gamma rays either through electromagnetic radiative corrections [7] or energy loss processes [8]; the final state $\mu^{+} \mu^{-}$produces $e^{+} e^{-}$by its decays. Thus the only possible invisible SM final states are neutrinos.

Of final states with neutrinos, we focus on $\bar{\nu} \nu$. Similar bounds could be derived for $\bar{\nu} \bar{\nu} \nu \nu$, but we assume that these are suppressed and/or that the convoluted Feynman diagrams required would contain charged particles, and hence gamma rays through (model-dependent) radiative corrections. Because of electroweak bremsstrahlung, final-state neutrinos are inevitably accompanied by weak bosons and hence gamma rays, primarily with $E_{\gamma} \simeq m_{\pi} / 2$; however, these gamma-ray constraints on $\left\langle\sigma_{A} v\right\rangle$ are weaker than or comparable to what we obtain directly with neutrinos [9].

To derive our bound on the total annihilation cross section, we assume $\operatorname{Br}(\bar{\nu} \nu) \simeq 100 \%$. This is not an assumption about realistic outcomes, but it is the right way to derive the most conservative upper bound for SM final states. Why is this a bound on the total cross section, and not just on the partial cross section to neutrinos? Suppose that $\operatorname{Br}(\bar{\nu} \nu)$ were reduced enough that the $1 / \operatorname{Br}(\bar{\nu} \nu)$ correction for an impure final state was necessary; at our factor-two precision, this occurs when another SM final state has a comparable branching ratio. For the total cross section set by the neutrino bound, any other pure final state would be more strongly constrained, thus making this cross section disallowed for all final states in the SM. Therefore, while setting this bound using neutrinos can be too conservative, it can never overreach.

Cosmic diffuse neutrinos: Signal. - The most direct approach to bound the $\chi \chi \rightarrow \bar{\nu} \nu$ cross section is to use the cosmic diffuse neutrino flux from dark matter annihilations in all halos in the universe as the signal. Since this is isotropic and time independent, it is challenging to detect above the background caused by the atmospheric neutrino flux. A complementary approach uses the Milky Way signals, which have somewhat different uncertainties on the predictions and data [10]. The data to test the diffuse signal are available in the full energy range now, but this is not yet true for all the directional signals. While the latter will likely be stronger eventually, going beyond our rough estimates will require proper experimental analyses.

The cosmic diffuse signal from $\chi \chi \rightarrow \bar{\nu} \nu$ annihilations depends on the radial density profile of each dark matter halo, the halo mass function (the relative weighting of halos of different masses), and how those halos evolve with redshift. We follow the calculations of Ullio et al. $[11,12]$; see also [13]. The signal spectrum is

$$
\frac{d \Phi_{\nu}}{d E}=\frac{\left\langle\sigma_{A} v\right\rangle}{2} \frac{c}{4 \pi H_{0}} \frac{\Omega_{\mathrm{DM}}^{2} \rho_{\text {crit }}^{2}}{m_{\chi}^{2}} \int_{0}^{z_{\text {up }}} d z \frac{\Delta^{2}(z)}{h(z)} \frac{d N_{\nu}\left(E^{\prime}\right)}{d E^{\prime}},
$$

where the $1 / 2$ is for assuming $\chi$ is its own antiparticle, $H_{0}=100 h \mathrm{~km} \mathrm{~s}^{-1} \mathrm{Mpc}^{-1}$ is the Hubble parameter, and $\Omega_{\mathrm{DM}}$ is the dark matter density in units of the critical density $\rho_{\text {crit }}$. We assume a flat universe, with $\Omega_{\mathrm{DM}}=0.3$, $\Omega_{\Lambda}=0.7, h=0.7$, and $h(z)=\left[(1+z)^{3} \Omega_{\mathrm{DM}}+\Omega_{\Lambda}\right]^{1 / 2}$.

The factor $\Delta^{2}(z)$ accounts for the increase in density due to the clustering of dark matter in halos, defined so that $\Delta^{2}=1$ corresponds to all dark matter being at its average density in the universe today. The concentration of halos and thus the value of $\Delta^{2}$ evolves with redshift. (Note that we have absorbed a factor of $(1+z)^{3}$ into the definition of $\Delta^{2}$, as in Ref. [12].) However, to collect most of the signal, we only need neutrino energies near $m_{\chi}$, and hence will be 
sensitive only to modest redshifts where it is accurate to take $\Delta^{2}(z) \simeq \Delta^{2}(0)$ [11]. Note that only $\Delta^{2}$ matters, and not its individual factors. The value of $\Delta^{2}$ does depend on the halo profile chosen. We adopt $\Delta^{2}=2 \times 10^{5}$ for Navarro, Frenk, and White (NFW) halos with moderate assumptions about the halo mass distribution. For cuspier Moore profiles, $\Delta^{2}$ could be $\simeq 10$ times larger, while for flatter Kravtsov profiles, it could be $\simeq 2$ times smaller; see the discussions in Ref. [10].

For $\chi \chi \rightarrow \bar{\nu} \nu$, the source spectrum $d N_{\nu} / d E^{\prime}$ is a delta function; neutrinos produced with energy $E^{\prime}$ are redshifted to the observed energy $E=E^{\prime} /(1+z)$, i.e.,

$$
\frac{d N_{\nu}\left(E^{\prime}\right)}{d E^{\prime}}=\frac{2}{3} \delta\left(m_{\chi}-E^{\prime}\right)=\frac{2}{3 E} \delta\left(z-\left(\frac{m_{\chi}}{E}-1\right)\right),
$$

where we have accounted for 2 neutrinos per annihilation, equally divided among 3 flavors. (Note that $\nu_{\mu}$ has a large fraction in every neutrino mass eigenstate, so any initial mix of mass or flavor eigenstates would be close to this.) In Fig. 2, we show example dark matter signals compared to the atmospheric neutrino background.

Using the neutrino signal, we can also derive a constraint from the relativistic energy density. Requiring $\Omega_{\text {rad }}<0.2$ at low redshift [14] leads to $\left\langle\sigma_{A} v\right\rangle<10^{-17} \times$ $\left(m_{\chi} / \mathrm{GeV}\right) \mathrm{cm}^{3} / \mathrm{s}$. While this bound applies to any light final state including non-SM particles such as purely sterile neutrinos, it is weak, and would require even greater halo modifications than the KKT model.

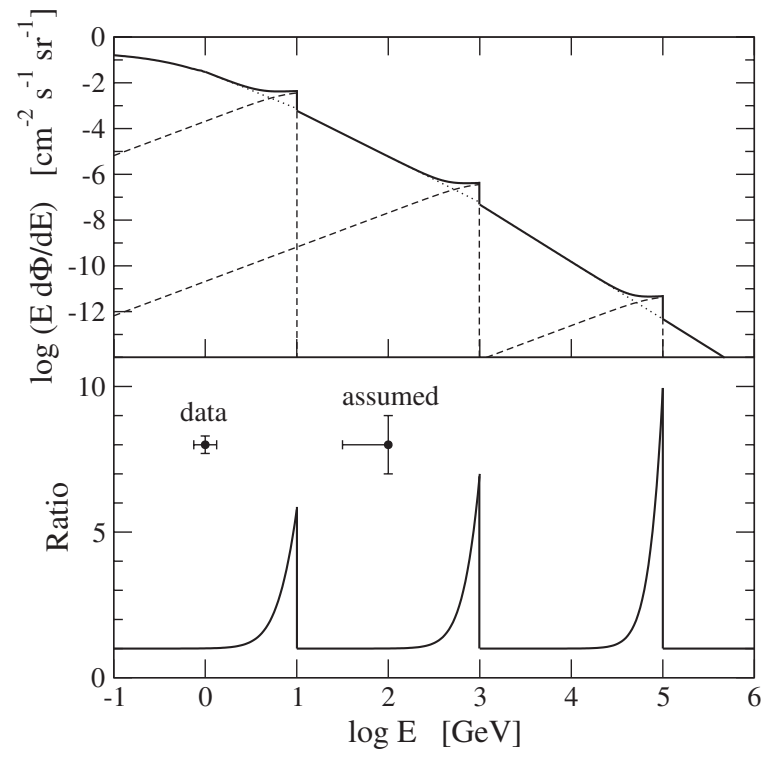

FIG. 2. Upper: Diffuse $\bar{\nu} \nu$ annihilation signal for $m_{\chi}=10$, $10^{3}$, and $10^{5} \mathrm{GeV}$, added to the atmospheric background, both as $\left(\bar{\nu}_{\mu}+\nu_{\mu}\right)$ and versus neutrino energy. As noted, the signals are most accurate for $E_{\nu} \geqslant m_{\chi} / 3$. Lower: Ratio of this sum and background. The $\left\langle\sigma_{A} v\right\rangle$ values at each example $m_{\chi}$ are chosen to be detectable by our conservative criteria; the data and assumed uncertainty scales are also indicated.
Cosmic diffuse neutrinos: Backgrounds. - How large of a neutrino signal is allowed by present data? As shown in Fig. 2, the signal spectrum is sharply peaked. To be insensitive to the spectrum shape, i.e., the redshift evolution, we define the signal as integrated over a bin of width $\Delta \log _{10} E_{\nu}=0.5$, just below $E_{\nu}=m_{\chi}$, (i.e., we consider $z \lesssim 2$, so we can ignore the tails at low energy). To be detectable, we require that the signal be $100 \%$ as large as the angle-averaged atmospheric neutrino $\left(\nu_{\mu}+\bar{\nu}_{\mu}\right)$ background, integrated in the same way. Signal and background are both somewhat smeared from received neutrino energy to detected energy, but well within this bin. This conservative approach allows us to simply derive the flux and annihilation cross section constraints over the very wide mass range $0.1-10^{5} \mathrm{GeV}$. We assume equal fluxes of $\left(\nu_{e}+\right.$ $\left.\bar{\nu}_{e}\right),\left(\nu_{\mu}+\bar{\nu}_{\mu}\right)$, and $\left(\nu_{\tau}+\bar{\nu}_{\tau}\right)$, any of which can be used to derive bounds.

The atmospheric neutrino $\left(\nu_{\mu}+\bar{\nu}_{\mu}\right)$ spectra as a function of neutrino energy have been derived from data from the Fréjus $\left(0.25-10^{4} \mathrm{GeV}\right.$, in 9 bins) [15] and AMANDA $\left(1.3 \times 10^{3}-3.0 \times 10^{5} \mathrm{GeV}\right.$, in 10 bins) [16] detectors. Neutrino attenuation in Earth will be significant only above $10^{5} \mathrm{GeV}$. The agreement with theoretical predictions against upward fluctuations in the data is very good, well below the $100 \%$ uncertainty that we adopted. These spectra were derived from neutrino-induced muon data by a regularized unfolding technique, which might miss a narrow signal.

We thus considered the data in more detail, finding that for $E_{\nu}=0.1-10^{4} \mathrm{GeV}$, such a signal is definitely excluded, especially using both the $\left(\nu_{\mu}+\bar{\nu}_{\mu}\right)$ and $\left(\nu_{e}+\right.$ $\bar{\nu}_{e}$ ) signals. The most useful data are from the SuperKamiokande detector. In Ref. [17], visible-energy spectra for each of $e$-like and $\mu$-like events from 0.1 to $100 \mathrm{GeV}$ are given in 4 log-spaced bins per decade. The agreement with predictions including neutrino oscillations is excellent; the moderate exceptions in some of the highestenergy bins are explainable [17]. Neutrinos with $E_{\nu} \sim$ $10-10^{3} \mathrm{GeV}$ are probed by the count rates (no spectra) of upward throughgoing muons [17], and similarly for $E_{\nu} \sim$ $10^{2}-10^{4} \mathrm{GeV}$ and upward showering muons [18]; both are also in excellent agreement with predictions.

Dedicated analyses of the measured data could improve the signal sensitivity by a factor 10-100, depending on the energy range. First, using the sharp feature in the spectrum at $m_{\chi}$, Fig. 2 shows that while the signal is comparable to the background when integrated over an energy bin, in the end-point region it is much larger. Second, the uncertainties below $10 \mathrm{GeV}$ are actually below $10 \%$, and apply to narrower bins in energy than we assumed [17]. Third, by $10 \mathrm{GeV}$, the $\left(\nu_{e}+\bar{\nu}_{e}\right)$ to $\left(\nu_{\mu}+\bar{\nu}_{\mu}\right)$ background flux ratio is $1 / 3$ and rapidly falling [19]; in addition, the $\left(\nu_{e}+\bar{\nu}_{e}\right)$ flux is strongly peaked at the horizon [19], while the dark matter signal is isotropic. Fourth, detailed analyses of Super-Kamiokande and AMANDA upward throughgoing 


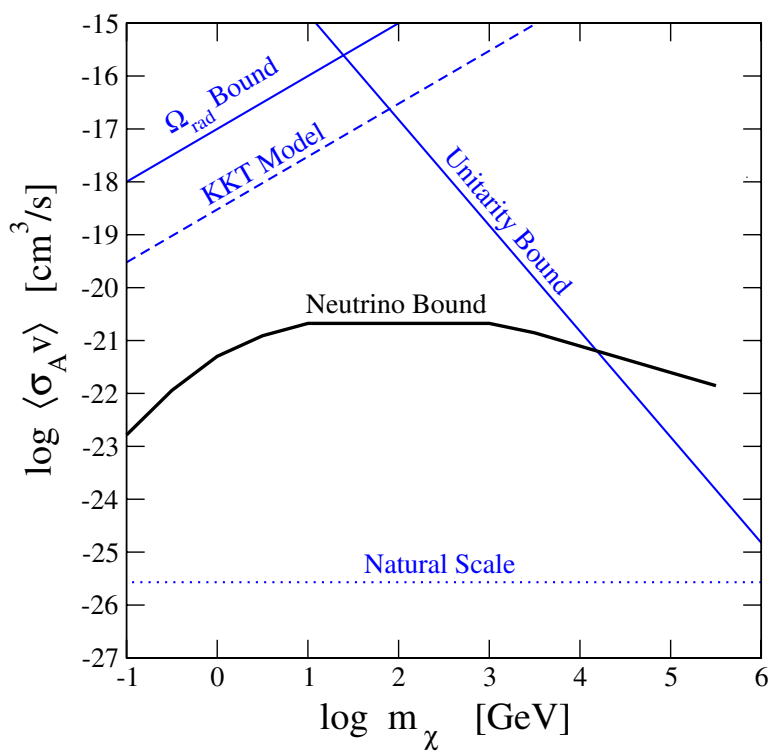

FIG. 3 (color online). Upper bounds on the dark matter total annihilation cross section in galaxy halos as a function of the dark matter mass, calculated as discussed in the text.

and upward showering muon data should be more sensitive than the simple count rates we used.

Conclusions. - We have shown that the dark matter total annihilation cross section in the late universe, i.e., the dark matter disappearance rate, can be directly and generally bounded by the least detectable SM states, i.e., the neutrino appearance rate. This can be simply and robustly constrained by comparing the diffuse signal from all dark matter halos to the terrestrial atmospheric neutrino background. Our bound on $\left\langle\sigma_{A} v\right\rangle$ is shown in Fig. 3. Over a large range in $m_{\chi}$, it is much stronger than the unitarity bound of Hui [3]. It strongly rules out the proposal of Kaplinghat, Knox, and Turner [4] to modify dark matter halos by annihilation. Our bound can be evaded with truly invisible non-SM final states. For a cross section above our bound, its ratio to our bound yields an upper limit on the branching ratio to $\mathrm{SM}$ final states required to invoke that large of a cross section.

Annihilation flattens halo cusps to cores of density $\rho_{A} \sim$ $m_{\chi} /\left(\left\langle\sigma_{A} v\right\rangle H_{0}^{-1}\right)$ [4]. Our bound implies that for all $m_{\chi} \gtrsim$ $0.1 \mathrm{GeV}$, this density is $\rho_{A} \gtrsim 5 \times 10^{3} \mathrm{GeV} / \mathrm{cm}^{3}$, which occurs only at radii $\leq 1 \mathrm{pc}$ in the Milky Way for an NFW profile. Annihilation should thus have minimal effects on galactic halos.

Detailed analyses by the Super-Kamiokande and AMANDA Collaborations should be able to improve our bound by a factor $10-100$ over the whole mass range. Halo substructure or mini-spikes around intermediate-mass black holes could increase the signal by orders of magnitude $[13,20]$. The sensitivity could thus become close to the natural scale for thermal relics, making it a new tool for testing even standard scenarios.
We are grateful to G. Bertone for very helpful discussions and collaboration on an early stage of this project. We thank S. Ando, S. Desai, M. Kachelriess, M. Kaplinghat, E. Komatsu, R. Scherrer, and H. Yüksel for helpful discussions. J.F. B. was supported by NSF CAREER Grant No. PHY-0547102; N.F. B. by the Sherman Fairchild Foundation through Caltech and by the Melbourne Research Grants Scheme; and G.D.M. by DOE Grant No. DE-FG02-91ER40690. We also thank CCAPP and OSU for support.

[1] G. Bertone, D. Hooper, and J. Silk, Phys. Rep. 405, 279 (2005).

[2] K. Griest and M. Kamionkowski, Phys. Rev. Lett. 64, 615 (1990).

[3] L. Hui, Phys. Rev. Lett. 86, 3467 (2001).

[4] M. Kaplinghat, L. Knox, and M. S. Turner, Phys. Rev. Lett. 85, 3335 (2000).

[5] A. Kusenko and P. J. Steinhardt, Phys. Rev. Lett. 87, 141301 (2001).

[6] D. N. Spergel and P. J. Steinhardt, Phys. Rev. Lett. 84, 3760 (2000).

[7] J.F. Beacom, N. F. Bell, and G. Bertone, Phys. Rev. Lett. 94, 171301 (2005); L. Bergstrom et al., Phys. Rev. Lett. 94, 131301 (2005).

[8] E. A. Baltz and L. Wai, Phys. Rev. D 70, 023512 (2004); D. P. Finkbeiner, arXiv:astro-ph/0409027.

[9] V. Berezinsky, M. Kachelriess, and S. Ostapchenko, Phys. Rev. Lett. 89, 171802 (2002); M. Kachelriess and P. D. Serpico, Phys. Rev. D 76, 063516 (2007).

[10] H. Yuksel, S. Horiuchi, J.F. Beacom, and S. Ando, arXiv:0707.0196.

[11] P. Ullio et al., Phys. Rev. D 66, 123502 (2002).

[12] L. Bergstrom, J. Edsjo, and P. Ullio, Phys. Rev. Lett. 87, 251301 (2001).

[13] J. E. Taylor and J. Silk, Mon. Not. R. Astron. Soc. 339, 505 (2003); K. Ahn and E. Komatsu, Phys. Rev. D 71, 021303(R) (2005); S. Ando, Phys. Rev. Lett. 94, 171303 (2005); S. Ando and E. Komatsu, Phys. Rev. D 73, 023521 (2006).

[14] A. R. Zentner and T. P. Walker, Phys. Rev. D 65, 063506 (2002).

[15] K. Daum et al., Z. Phys. C 66, 417 (1995).

[16] J. Ahrens et al., Phys. Rev. D 66, 012005 (2002); K. Münich et al., arXiv:astro-ph/0509330.

[17] Y. Ashie et al., Phys. Rev. D 71, 112005 (2005).

[18] S. Desai, Ph.D. thesis, Boston University, 2004; S. Desai et al., in Proceedings of the 28th International Cosmic Ray Conference, edited by T. Kajita et al. (Tsukuba, Japan, 2003), p. 1673.

[19] T. K. Gaisser and M. Honda, Annu. Rev. Nucl. Part. Sci. 52, 153 (2002); M. Honda et al., Phys. Rev. D 70, 043008 (2004).

[20] G. Bertone, A. R. Zentner, and J. Silk, Phys. Rev. D 72, 103517 (2005); G. Bertone, Phys. Rev. D 73, 103519 (2006); S. Horiuchi and S. Ando, arXiv:astro-ph/0607042. 